\title{
The spectrum of the twisted Dirac operator on Kähler submanifolds of the complex projective space
}

\author{
Nicolas Ginoux* and Georges Habib ${ }^{\dagger}$
}

January 25, 2011

\begin{abstract}
We establish an upper estimate for the small eigenvalues of the twisted Dirac operator on Kähler submanifolds in Kähler manifolds carrying Kählerian Killing spinors. We then compute the spectrum of the twisted Dirac operator of the canonical embedding $\mathbb{C} P^{d} \rightarrow \mathbb{C} P^{n}$ in order to test the sharpness of the upper bounds.
\end{abstract}

\section{Introduction}

One of the basic tools to get upper bounds for the eigenvalues of the twisted Dirac operator on spin submanifolds is the min-max principle. The idea consists in computing in terms of geometric quantities the so-called Rayleigh-quotient applied to some test section coming from the ambient manifold. In [1], C. Bär established with the help of the min-max principle upper eigenvalue estimates for submanifolds in $\mathbb{R}^{n+1}, \mathbb{S}^{n+1}$ and $\mathbb{H}^{n+1}$, estimate which is sharp in the first two cases. In the same spirit, the first-named author studied in his $\mathrm{PhD}$ thesis [6] different situations where the ambient manifold admits natural testspinors carrying geometric information.

In this paper, we consider a closed spin Kähler submanifold $M$ of a Kähler spin manifold $\widetilde{M}$ and derive upper bounds for the small eigenvalues of the corresponding twisted Dirac operator in case $\widetilde{M}$ carries so-called Kählerian Killing spinors (see (2.3) for a definition). Interestingly enough, the upper bound turns out to depend only on the complex dimension of $M$ (Theorem 2.2). Whether this estimate is sharp is a much more involved question. A first approach consists in finding lower bounds for the spectrum and to compare them with the upper ones. In Section 3, we prove a Kirchberg-type lower bound for the eigenvalues of any twisted Dirac operator on a closed Kähler manifold (Corollary 3.2). Here the curvature of the twisting bundle has to be involved. Even for the canonical embedding $\mathbb{C} P^{d} \rightarrow \mathbb{C} P^{n}$, the presence of that normal curvature does not allow to state the equality between the lower bound and the upper one, see Proposition 3.3. The next approach consists in computing explicitly the spectrum of the twisted Dirac operator, at least for particular embeddings. In Section 4 , we determine the eigenvalues (with multiplicities) of the twisted Dirac operator of the canonical embedding $\mathbb{C} P^{d} \rightarrow \mathbb{C} P^{n}$, using earlier results by M. Ben Halima [3]. We first remark that the spinor bundle of the normal bundle splits into a direct sum of powers of the tautological bundle (Corollary 4.4). We deduce the spectrum of the twisted Dirac operator in Theorem 4.8, where we also include the multiplicities with the help of Weyl's character formula. We conclude that, for $d<\frac{n+1}{2}$, the twisted Dirac operator admits 0 as a lowest eigenvalue and $(n+1)(2 d+1-n)$ for $d \geq \frac{n+1}{2}$ (see Proposition 4.9). This implies that, for $d=1$, the upper estimate is optimal for $n=3,5,7$, however it is no more optimal for $n \geq 9$.

This work is partially based on and extends the first-named author's $\mathrm{PhD}$ thesis [6, Ch. 4].

Acknowledgment. We thank the Max-Planck Institute for Mathematics in the Sciences and the University of Regensburg for their support.

\footnotetext{
*Fakultät für Mathematik, Universität Regensburg, $\quad$ D-93040 Regensburg, nicolas.ginoux@mathematik. uni-regensburg.de

$\dagger$ Lebanese University, Faculty of Sciences II, Department of Mathematics, P.O. Box 90656 Fanar-Matn, Lebanon, E-mail: ghabib@ul.edu.lb
} 


\section{Upper bounds for the submanifold Dirac operator of a Kähler submanifold}

In this section, we prove a priori upper bounds for the smallest eigenvalues of some twisted Dirac operator on complex submanifolds in Kähler manifolds admitting so-called Kählerian Killing spinors.

Let $M^{2 d}$ be an immersed almost-complex submanifold in a Kähler manifold $\left(\widetilde{M}^{2 n}, g, J\right)$ ( "almost-complex" means that $J(T M)=T M)$. Then for the induced metric and almost-complex structure the manifold $\left(M^{2 d}, g, J\right)$ is Kähler, in particular its immersion is minimal in $\left(\widetilde{M}^{2 n}, g, J\right)$. We denote by $\widetilde{\Omega}, \Omega$ and $\Omega_{N}$ the Kähler form of $\left(\widetilde{M}^{2 n}, g, J\right),\left(M^{2 d}, g, J\right)$ and of the normal bundle $N M \longrightarrow M$ of the immersion respectively (in our convention, $\Omega(X, Y)=g(J(X), Y)$ for all $X, Y)$.

Assuming both $\left(M^{2 d}, g, J\right)$ and $\left(\widetilde{M}^{2 n}, g, J\right)$ to be spin, the bundle $N M$ carries an induced spin structure such that the restricted (complex) spinor bundle $\Sigma \widetilde{M}_{\left.\right|_{M}}$ of $\widetilde{M}$ can be identified with $\Sigma M \otimes \Sigma N$, where $\Sigma M$ and $\Sigma N$ are the spinor bundles of $M$ and $N M$ respectively. Denote by "."., "." and "."the Clifford multiplications of $M, N M$ and $\widetilde{M}$ respectively. By a suitable choice of invariant Hermitian inner product $\langle\cdot, \cdot\rangle$ (with associated norm $|\cdot|$ ) on $\Sigma \widetilde{M}$ the identification above can be made unitary. Moreover, it can be assumed to respect the following rules: given any $X \in T M$ and $\nu \in N M$, one has

$$
\begin{aligned}
X \cdot \varphi & =\left\{X \dot{M} \otimes\left(\operatorname{Id}_{\Sigma^{+} N}-\operatorname{Id}_{\Sigma^{-} N}\right)\right\} \varphi \\
\nu \cdot \varphi & =\left(\operatorname{Id} \otimes \nu_{N}\right) \varphi
\end{aligned}
$$

for all $\varphi \in \Sigma \widetilde{M}_{\left.\right|_{M}}=\Sigma M \otimes \Sigma N$. Here $\Sigma N=\Sigma^{+} N \oplus \Sigma^{-} N$ stands for the orthogonal and parallel splitting induced by the complex volume form, see e.g. [6, Sec. 1.2.1] or [9, Sec. 2.1]. The following Gauss-type formula holds for the spinorial Levi-Civita connections $\widetilde{\nabla}$ and $\nabla:=\nabla^{\Sigma M \otimes \Sigma N}$ on $\Sigma \widetilde{M}$ and $\Sigma M \otimes \Sigma N$ respectively: for all $X \in T M$ and $\varphi \in \Gamma\left(\Sigma \widetilde{M}_{\left.\right|_{M}}\right)$,

$$
\widetilde{\nabla}_{X} \varphi=\nabla_{X} \varphi+\frac{1}{2} \sum_{j=1}^{2 d} e_{j} \cdot I I\left(X, e_{j}\right) \cdot \varphi,
$$

where $\left(e_{j}\right)_{1 \leq j \leq 2 d}$ is any local orthonormal basis of $T M$ and $I I$ the second fundamental form of the immersion.

Recall that, for a complex constant $\alpha$, an $\alpha$-Kählerian Killing spinor on a Kähler spin manifold $\left(\widetilde{M}^{2 n}, g, J\right)$ is a pair $(\psi, \phi)$ of spinors satisfying, for all $X \in T \widetilde{M}$,

$$
\mid \begin{aligned}
& \widetilde{\nabla}_{X} \psi=-\alpha p_{-}(X) \cdot \phi \\
& \widetilde{\nabla}_{X} \phi=-\alpha p_{+}(X) \cdot \psi
\end{aligned}
$$

where $p_{ \pm}(X):=\frac{1}{2}(X \mp i J(X))$. The existence of a non-zero $\alpha$-Kählerian Killing spinor on $\left(\widetilde{M}^{2 n}, g, J\right)$ imposes the metric to be Einstein with scalar curvature $\widetilde{S}=4 n(n+1) \alpha^{2}$ (in particular $\alpha$ must be either real or purely imaginary), the complex dimension $n$ of $\widetilde{M}$ to be odd and the spinors $\psi, \phi$ to lie in particular eigenspaces of the Clifford action of $\widetilde{\Omega}$, namely

$$
\mid \begin{array}{ll}
\widetilde{\Omega} \cdot \psi & =-i \psi \\
\widetilde{\Omega} \cdot \phi & =i \phi
\end{array}
$$

Actually a Kähler spin manifold carries a non-zero $\alpha$-Kählerian Killing spinor with $\alpha \in \mathbb{R}^{\times}$if and only if it is the twistor-space of a quaternionic-Kähler manifold with positive scalar curvature (in particular it must be $\mathbb{C P}^{n}$ if $n \equiv 1(4)$ ), see [12. For purely imaginary $\alpha$ only partial results are known, the prominent examples being the complex hyperbolic space [10, Thm. 13] as well as doubly-warped products associated to some circle bundles over hyperkähler manifolds 8 .

We need the following lemma [6, Lemme 4.4]: 
Lemma 2.1 Let $\left(M^{2 d}, g, J\right)$ be a Kähler spin submanifold of a Kähler spin manifold $\left(\widetilde{M}^{2 n}, g, J\right)$ and assume the existence of an $\alpha$-Kählerian Killing spinor $(\psi, \phi)$ on $\left(\widetilde{M}^{2 n}, g, J\right)$. Then

$$
\left(D_{M}^{\Sigma N}\right)^{2}(\psi+\phi)=(d+1)^{2} \alpha^{2}(\psi+\phi)+\alpha^{2} \Omega_{N} \cdot \Omega_{N} \cdot(\psi+\phi) .
$$

Proof: Fix a local orthonormal basis $\left(e_{j}\right)_{1 \leq j \leq 2 n}$ of $T \widetilde{M}_{\left.\right|_{M}}$ with $e_{j} \in T M$ for all $1 \leq j \leq 2 d$ and $e_{j} \in N M$ for all $2 d+1 \leq j \leq 2 n$. Introduce the auxiliary Dirac-type operator $\widehat{D}:=\sum_{j=1}^{2 d} e_{j} \cdot \widetilde{\nabla}_{e_{j}}: \Gamma\left(\Sigma \widetilde{M}_{\left.\right|_{M}}\right) \longrightarrow$ $\Gamma\left(\Sigma \widetilde{M}_{\left.\right|_{M}}\right)$. As a consequence of the Gauss-type formula (2.2), the operators $\widehat{D}^{2}$ and $\left(D_{M}^{\Sigma N}\right)^{2}$ are related by [6. Lemme 4.1]

$$
\widehat{D}^{2} \varphi=\left(D_{M}^{\Sigma N}\right)^{2} \varphi-d^{2}|H|^{2} \varphi-d \sum_{j=1}^{2 d} e_{j} \cdot \nabla_{e_{j}}^{N} H \cdot \varphi,
$$

where $H:=\frac{1}{2 d} \operatorname{tr}(I I)$ is the mean curvature vector field of the immersion. In particular $\widehat{D}^{2}$ and $\left(D_{M}^{\Sigma N}\right)^{2}$ coincide as soon as the mean curvature vector field of the immersion vanishes, condition which is fulfilled here. Using $\sum_{j=1}^{2 n} p_{+}\left(e_{j}\right) \cdot p_{-}\left(e_{j}\right)=i \widetilde{\Omega}-n$ and $\sum_{j=1}^{2 n} p_{-}\left(e_{j}\right) \cdot p_{+}\left(e_{j}\right)=-i \widetilde{\Omega}-n$, we compute:

$$
\begin{aligned}
\widehat{D} \psi & =\sum_{j=1}^{2 d} e_{j} \cdot \widetilde{\nabla}_{e_{j}} \psi \\
\stackrel{\underline{2.31}}{=} & -\alpha \sum_{j=1}^{2 d} e_{j} \cdot p_{-}\left(e_{j}\right) \cdot \phi \\
& =-\alpha \sum_{j=1}^{2 d} p_{+}\left(e_{j}\right) \cdot p_{-}\left(e_{j}\right) \cdot \phi \\
& =-\alpha(i \Omega \cdot-d) \phi \\
& =-\alpha(i \widetilde{\Omega} \cdot-d) \phi+i \alpha \Omega_{N} \cdot \phi \\
\stackrel{2.4}{=} & (d+1) \alpha \phi+i \alpha \Omega_{N} \cdot \phi .
\end{aligned}
$$

Similarly,

$$
\begin{aligned}
\widehat{D} \phi & =\sum_{j=1}^{2 d} e_{j} \cdot \widetilde{\nabla}_{e_{j}} \phi \\
\stackrel{(2.3)}{=} & -\alpha \sum_{j=1}^{2 d} e_{j} \cdot p_{+}\left(e_{j}\right) \cdot \psi \\
& =-\alpha \sum_{j=1}^{2 d} p_{-}\left(e_{j}\right) \cdot p_{+}\left(e_{j}\right) \cdot \psi \\
& =-\alpha(-i \Omega \cdot-d) \psi \\
& =
\end{aligned}
$$

so that

$$
\widehat{D}(\psi+\phi)=(d+1) \alpha(\psi+\phi)+i \alpha \Omega_{N} \cdot(\phi-\psi) .
$$

To compute $\widehat{D}^{2}(\psi+\phi)$ we need the commutator of $\Omega_{N} \cdot$ with $\widehat{D}$. For any $\varphi \in \Gamma\left(\Sigma \widetilde{M}_{\left.\right|_{M}}\right)$, one has

$$
\begin{aligned}
\widehat{D}\left(\Omega_{N} \cdot \varphi\right) & =\sum_{j=1}^{2 d} e_{j} \cdot \widetilde{\nabla}_{e_{j}}\left(\Omega_{N} \cdot \varphi\right) \\
& =\sum_{j=1}^{2 d} e_{j} \cdot \widetilde{\nabla}_{e_{j}} \Omega_{N} \cdot \varphi+e_{j} \cdot \Omega_{N} \cdot \widetilde{\nabla}_{e_{j}} \varphi
\end{aligned}
$$




$$
\begin{aligned}
& =\sum_{j=1}^{2 d} \Omega_{N} \cdot e_{j} \cdot \widetilde{\nabla}_{e_{j}} \varphi+e_{j} \cdot \widetilde{\nabla}_{e_{j}} \Omega_{N} \cdot \varphi \\
& =\Omega_{N} \cdot \widehat{D} \varphi+\sum_{j=1}^{2 d} e_{j} \cdot \widetilde{\nabla}_{e_{j}} \Omega_{N} \cdot \varphi
\end{aligned}
$$

with, for all $X, Y \in T M$ and $\nu \in N M$,

$$
\begin{aligned}
\left(\widetilde{\nabla}_{X} \Omega_{N}\right)(Y, \nu) & =-\Omega_{N}\left(\widetilde{\nabla}_{X} Y, \nu\right) \\
& =-g\left(J\left(\widetilde{\nabla}_{X} Y\right), \nu\right) \\
& =-g(J(I I(X, Y)), \nu),
\end{aligned}
$$

so that

$$
\begin{aligned}
\sum_{j=1}^{2 d} e_{j} \cdot \widetilde{\nabla}_{e_{j}} \Omega_{N} \cdot \varphi & =-\sum_{j, k=1}^{2 d} \sum_{l=2 d+1}^{2 n} g\left(J\left(I I\left(e_{j}, e_{k}\right)\right), e_{l}\right) e_{j} \cdot e_{k} \cdot e_{l} \cdot \varphi \\
& =-\sum_{j, k=1}^{2 d} e_{j} \cdot e_{k} \cdot J\left(I I\left(e_{j}, e_{k}\right)\right) \cdot \varphi \\
& =\sum_{j=1}^{2 d} J\left(I I\left(e_{j}, e_{j}\right)\right) \cdot \varphi \\
& =0,
\end{aligned}
$$

since the immersion is minimal. Hence $\widehat{D}\left(\Omega_{N} \cdot \varphi\right)=\Omega_{N} \cdot \widehat{D} \varphi$ and we deduce that

$$
\begin{aligned}
\widehat{D}^{2}(\psi+\phi) & =(d+1) \alpha \widehat{D}(\psi+\phi)+i \alpha \widehat{D}\left(\Omega_{N} \cdot(\phi-\psi)\right) \\
& =(d+1)^{2} \alpha^{2}(\psi+\phi)+i(d+1) \alpha^{2} \Omega_{N} \cdot(\phi-\psi)+i \alpha \Omega_{N} \cdot \widehat{D}(\phi-\psi) \\
& =(d+1)^{2} \alpha^{2}(\psi+\phi)+i(d+1) \alpha^{2} \Omega_{N} \cdot(\phi-\psi)+i \alpha \Omega_{N} \cdot\left((d+1) \alpha(\psi-\phi)-i \alpha \Omega_{N} \cdot(\psi+\phi)\right) \\
& =(d+1)^{2} \alpha^{2}(\psi+\phi)+\alpha^{2} \Omega_{N} \cdot \Omega_{N} \cdot(\psi+\phi),
\end{aligned}
$$

which concludes the proof.

Next we formulate the main theorem of this section. Its proof requires some further notations. Given any rank- $2 k$-Hermitian spin bundle $E \longrightarrow M$ with metric connection preserving the complex structure, the Clifford action of the Kähler form $\Omega_{E}$ of $E$ splits the spinor bundle $\Sigma E$ of $E$ into the orthogonal and parallel sum

$$
\Sigma E=\bigoplus_{r=0}^{k} \Sigma_{r} E
$$

where $\Sigma_{r} E:=\operatorname{Ker}\left(\Omega_{E} \cdot-i(2 r-k) \mathrm{Id}\right)$ is a subbundle of complex $\operatorname{rank}\left(\begin{array}{c}k \\ r\end{array}\right)$. Moreover, given any $V \in E$, one has $p_{ \pm}(V) \cdot \Sigma_{r} E \subset \Sigma_{r \pm 1} E$.

Theorem 2.2 (see [6, Thm. 4.2]) Let $\left(M^{2 d}, g, J\right)$ be a closed Kähler spin submanifold of a Kähler spin manifold $\left(\widetilde{M}^{2 n}, g, J\right)$ and consider the induced spin structure on the normal bundle. Assume the existence of a complex $\mu$-dimensional space of non-zero $\alpha$-Kählerian Killing spinor on $\left(\widetilde{M}^{2 n}, g, J\right)$ for some $\alpha \in \mathbb{R}^{\times}$. Then there are $\mu$ eigenvalues $\lambda$ of $\left(D_{M}^{\Sigma N}\right)^{2}$ satisfying

$$
\lambda \leq \begin{cases}(d+1)^{2} \alpha^{2} & \text { if } d \text { is odd } \\ d(d+2) \alpha^{2} & \text { if } d \text { is even } .\end{cases}
$$

If moreover (2.7) is an equality for the smallest eigenvalue $\lambda$ and some odd $d$, then $\sum_{j=1}^{2 d} e_{j} \cdot I I\left(X, e_{j}\right) \cdot \psi=$ $\sum_{j=1}^{2 d} e_{j} \cdot I I\left(X, e_{j}\right) \cdot \phi=0$. 
Proof: Let $(\psi, \phi)$ be a non-zero $\alpha$-Kählerian Killing spinor on $\left(\widetilde{M}^{2 n}, g, J\right)$. We evaluate the Rayleighquotient $\frac{\int_{M}\left\langle\left(D_{M}^{\Sigma N}\right)^{2}(\psi+\phi), \psi+\phi\right\rangle v_{g}}{\int_{M}\langle\psi+\phi, \psi+\phi\rangle v_{g}}$ and apply the min-max principle. It can be deduced from Lemma 2.1 that

$$
\begin{aligned}
\left\langle\left(D_{M}^{\Sigma N}\right)^{2}(\psi+\phi), \psi+\phi\right\rangle & =(d+1)^{2} \alpha^{2}|\psi+\phi|^{2}+\alpha^{2}\left\langle\Omega_{N} \cdot \Omega_{N} \cdot(\psi+\phi), \psi+\phi\right\rangle \\
& =(d+1)^{2} \alpha^{2}|\psi+\phi|^{2}-\alpha^{2}\left|\Omega_{N} \cdot(\psi+\phi)\right|^{2}
\end{aligned}
$$

Using (2.6) for $E=N M$ we observe that $\left|\Omega_{N} \cdot(\psi+\phi)\right| \geq|\psi+\phi|$ if $n-d$ is odd (i.e., if $d$ is even) and is nonnegative otherwise. The inequality follows.

If $d$ is odd and (2.7) is an equality for the smallest eigenvalue, then $\left(D_{M}^{\Sigma N}\right)^{2}(\psi+\phi)=(d+1)^{2} \alpha^{2}(\psi+\phi)$ and $\Omega_{N} \cdot(\psi+\phi)=0$. Since $\widetilde{\Omega}=\Omega \oplus \Omega_{N}$ one has $\Sigma_{r} \widetilde{M}_{\left.\right|_{M}}=\bigoplus_{s=0}^{r} \Sigma_{s} M \otimes \Sigma_{r-s} M$ (where each component vanishes as soon as the index exceeds its allowed bounds), so that $\psi \in \Gamma\left(\Sigma_{\frac{d-1}{2}} M \otimes \Sigma_{\frac{n-d}{2}} N\right)$ and $\phi \in \Gamma\left(\Sigma_{\frac{d+1}{2}} M \otimes \Sigma_{\frac{n-d}{2}} N\right)$. Coming back to the Gauss-type equation (2.2), one obtains

$$
\mid \begin{array}{cc}
\nabla_{X} \psi & =-\alpha p_{-}(X) \cdot \phi-\frac{1}{2} \sum_{j=1}^{2 d} e_{j} \cdot I I\left(X, e_{j}\right) \cdot \psi \\
\nabla_{X} \phi & =-\alpha p_{+}(X) \cdot \psi-\frac{1}{2} \sum_{j=1}^{2 d} e_{j} \cdot I I\left(X, e_{j}\right) \cdot \phi
\end{array}
$$

for all $X \in T M$. Looking more precisely at the components of each side of those identities, one notices that, pointwise, $\nabla_{X} \psi \in \Sigma_{\frac{d-1}{2}} M \otimes \Sigma_{\frac{n-d}{2}} N$ and, using (2.1), that $p_{-}(X) \cdot \phi \in \Sigma_{\frac{d-1}{2}} M \otimes \Sigma_{\frac{n-d}{2}} N$. But pointwise $\sum_{j=1}^{2 d} e_{j} \cdot I I\left(X, e_{j}\right) \cdot \psi \in\left(\Sigma_{\frac{d-3}{2}} M \otimes \Sigma_{\frac{n-d-2}{2}} N\right) \oplus\left(\Sigma_{\frac{d-3}{2}} M \otimes \Sigma_{\frac{n-d+2}{2}} N\right) \oplus\left(\Sigma_{\frac{d+1}{2}} M \otimes \Sigma_{\frac{n-d-2}{2}} N\right) \oplus$ $\left(\sum_{\frac{d+1}{2}} M \otimes \Sigma_{\frac{n-d+2}{2}} N\right)$, in particular this term must vanish. Analogously one has $\sum_{j=1}^{2 d} e_{j} \cdot I I\left(X, e_{j}\right) \cdot \phi=0$. This concludes the proof.

To test the sharpness of the estimate (2.7), we would like to first compare it to an a priori lower bound. This is the object of the next section.

\section{$3 \quad$ Kirchberg-type lower bounds}

In this section, we aim at giving Kirchberg type estimates for any twisted Dirac operator on closed Kähler spin manifolds. First consider a Kähler spin manifold $M$ of complex dimension $d$ and let $E$ be any rank $2 k$-vector bundle over $M$ endowed with a metric connection. We define a connection on the vector bundle $\Sigma:=\Sigma M \otimes E$ by $\nabla:=\nabla^{\Sigma M \otimes E}$. The Dirac operator of $M$ twisted with $E$ is defined by $D_{M}^{E}: \Gamma(\Sigma) \rightarrow \Gamma(\Sigma), D_{M}^{E}:=\sum_{i=1}^{2 d} e_{i} \cdot \nabla_{e_{i}}$, where $\left\{e_{i}\right\}_{1 \leq i \leq 2 d}$ is any local orthonormal basis of $T M$ and "." stands for the Clifford multiplication tensorized with the identity of $E$. The square of the Dirac-type operator $D_{M}^{E}$ is related to the rough Laplacian via the following Schrödinger-Lichnerowicz formula [11, Thm. II.8.17]

$$
\left(D_{M}^{E}\right)^{2}=\nabla^{*} \nabla+\frac{1}{4}\left(\mathrm{Scal}_{M}+R^{E}\right)
$$

where $\mathrm{Scal}_{M}$ denotes the scalar curvature of $M$ and $R^{E}$ is the endomorphism tensor field given by

$$
\begin{aligned}
R^{E}: \Sigma & \longrightarrow \Sigma \\
\psi & \longmapsto 2 \sum_{i, j=1}^{2 d}\left(e_{i} \cdot e_{j} \cdot \operatorname{Id} \otimes R_{e_{i}, e_{j}}^{E}\right) \psi .
\end{aligned}
$$

Recall that for any eigenvalue $\lambda$ of the Dirac operator, there exists an eigenspinor $\varphi$ associated with $\lambda$ such that $\varphi=\varphi_{r}+\varphi_{r+1}$, where $\varphi_{r}$ is a section in $\Sigma_{r}:=\Sigma_{r} M \otimes E$. Here $\Sigma_{r} M$ is the subundle $\operatorname{Ker}(\Omega \cdot-i(2 r-d) \operatorname{Id})$ of $\Sigma M$. Such an eigenspinor $\varphi$ is called of type $(r, r+1)$. In order to estimate the eigenvalues of the twisted Dirac operator we define, as in the classical way, on each subbundle $\Sigma_{r}$ the twisted twistor operator for all $X \in \Gamma(T M), \psi_{r} \in \Sigma_{r}$ by [5]

$$
P_{X} \psi_{r}:=\nabla_{X} \psi_{r}+a_{r} p_{-}(X) \cdot D_{+} \psi_{r}+b_{r} p_{+}(X) \cdot D_{-} \psi_{r},
$$

where $a_{r}=\frac{1}{2(r+1)}, b_{r}=\frac{1}{2(m-r+1)}$ and $D_{ \pm} \psi_{r}=\sum_{i=1}^{2 d} p_{ \pm}\left(e_{i}\right) \cdot \nabla_{e_{i}} \psi_{r}$.

We state the following lemma: 
Lemma 3.1 For any eigenspinor $\varphi$ of type $(r, r+1)$, we have the following inequalities

$$
\lambda^{2} \geq\left\{\begin{array}{l}
\frac{1}{4\left(1-a_{r}\right)} \inf _{M_{\varphi_{r}}}\left(\mathrm{Scal}_{M}+R_{\varphi_{r}}^{E}\right), \\
\frac{1}{4\left(1-b_{r+1}\right)} \inf _{M_{\varphi_{r+1}}}\left(\mathrm{Scal}_{M}+R_{\varphi_{r+1}}^{E}\right),
\end{array}\right.
$$

where $R_{\phi}^{E}:=\Re\left(R^{E}(\phi), \frac{\phi}{|\phi|^{2}}\right)$ is defined on the set $M_{\phi}=\{x \in M \mid \phi(x) \neq 0\}$ for all spinor $\phi \in \Sigma$.

Proof: Using the identity $\sum_{i=1}^{2 d} e_{i} \cdot P_{e_{i}} \psi_{r}=0$, one can easily prove by a straightforward computation that for any spinor $\psi_{r} \in \Sigma_{r}$

$$
\left|P \psi_{r}\right|^{2}=\left|\nabla \psi_{r}\right|^{2}-a_{r}\left|D_{+} \psi_{r}\right|^{2}-b_{r}\left|D_{-} \psi_{r}\right|^{2} .
$$

Applying Equation (3.2) to $\varphi_{r}$ and $\varphi_{r+1}$ respectively and integrating over $M$, we get with the use of the Schrödinger-Lichnerowicz formula that

$$
0 \leq \int_{M}\left[\lambda^{2}\left(1-a_{r}\right)-\frac{1}{4}\left(\operatorname{Scal}_{M}+R_{\varphi_{r}}^{E}\right)\right]\left|\varphi_{r}\right|^{2} .
$$

Also that,

$$
0 \leq \int_{M}\left[\lambda^{2}\left(1-b_{r+1}\right)-\frac{1}{4}\left(\operatorname{Scal}_{M}+R_{\varphi_{r+1}}^{E}\right)\right]\left|\varphi_{r+1}\right|^{2},
$$

from which the proof of the lemma follows.

One can get rid of the dependence of the eigenspinors $\varphi_{r}$ and $\varphi_{r+1}$ in the r.h.s. of (3.1):

Corollary 3.2 Let $\kappa_{1}$ be the smallest eigenvalue of the (pointwise) self-adjoint operator $R^{E}$. Then

$$
\lambda^{2} \geq \begin{cases}\frac{d+1}{4 d}\left(\mathrm{Scal}_{0}+\kappa_{1}\right) & \text { if } d \text { is odd } \\ \frac{d}{4(d-1)}\left(\mathrm{Scal}_{0}+\kappa_{1}\right) & \text { if } d \text { is even },\end{cases}
$$

where $\mathrm{Scal}_{0}$ denotes the infimum of the scalar curvature.

Proof: Let us choose the lowest integer $r \in\{0,1, \cdots, d\}$ such that $\varphi$ is of type $(r, r+1)$. The existence of anti-linear parallel maps on $\Sigma M$ commuting with the Clifford multiplication (see e.g. [7, Lemma 1]) allows to impose that $r \leq \frac{d-1}{2}$ if $d$ is odd and $r \leq \frac{d-2}{2}$ if $d$ is even. This concludes the proof.

In the following, we formulate the estimates (3.1) for the situation where $M$ is a complex submanifold of the projective space $\mathbb{C P}^{n}$ and $E$ is the spinor bundle of the normal bundle $N M$ of the immersion. To do this, we will estimate $R_{\phi}^{E}$ for all spinor field $\phi \in \Sigma$ in terms of the second fundamental form of the immersion.

Proposition 3.3 Let $\left(M^{2 d}, g, J\right)$ be a Kähler spin submanifold of the projective space $\mathbb{C P}^{n}$. For all spinor field $\phi \in \Sigma$, the curvature is equal to

$$
R_{\phi}^{E}=-4 \Re\left(\Omega \cdot \Omega_{N} \cdot \phi, \frac{\phi}{|\phi|^{2}}\right)-\sum_{i, j, p=1}^{2 d} \Re\left(e_{i} \cdot e_{j} \cdot I I\left(e_{i}, e_{p}\right) \cdot I I\left(e_{j}, e_{p}\right) \cdot \phi, \frac{\phi}{|\phi|^{2}}\right)+|I I|^{2} .
$$

where $\Omega$ is the Kähler form of $M$.

Proof: First, recall that for all $X, Y \in \Gamma(T M)$ and $U, V$ sections in $N M$, the normal curvature is related to the one of $\mathbb{C P}^{n}$ via the formula [4, Thm. 1.1.72]

$$
\begin{aligned}
\left(R_{X, Y}^{N M} U, V\right)= & \left(R_{X, Y}^{\mathbb{C P}^{n}} U, V\right)-\left(B_{X} U, B_{Y} V\right)+\left(B_{Y} U, B_{X} V\right) \\
= & 2 g(X, J(Y)) g(J(U), V)-\sum_{p=1}^{2 d} g\left(I I\left(X, e_{p}\right), U\right) g\left(I I\left(Y, e_{p}\right), V\right) \\
& +\sum_{p=1}^{2 d} g\left(I I\left(Y, e_{p}\right), U\right) g\left(I I\left(X, e_{p}\right), V\right),
\end{aligned}
$$


where $B_{X}: N M \rightarrow T M$ is the tensor field defined by $g\left(B_{X} U, Y\right)=-g(I I(X, Y), U)$ and $\left\{e_{p}\right\}_{1 \leq p \leq 2 d}$ is a local orthonormal basis of $T M$. Here we used the fact that the curvature of $\mathbb{C P}^{n}$ is given for all $X, Y, Z \in T \mathbb{C P}^{n}$ by

$$
R_{X, Y}^{\mathbb{C P} Z} Z=(X \wedge Y+J X \wedge J Y+2 g(X, J Y) J) Z
$$

with $(X \wedge Y) Z=g(Y, Z) X-g(X, Z) Y$. Hence by (3.4), the normal spinorial curvature associated with any spinor field $\phi$ is then equal to

$$
\begin{aligned}
R_{e_{i}, e_{j}}^{E} \phi & =\frac{1}{4} \sum_{k, l=1}^{2(n-d)} g\left(R_{e_{i}, e_{j}}^{N M} e_{k}, e_{l}\right) e_{k} \cdot e_{l} \cdot \phi \\
& =\frac{1}{2} \sum_{k=1}^{2(n-d)} g\left(e_{i}, J\left(e_{j}\right)\right) e_{k} \cdot J e_{k} \cdot \phi-\frac{1}{2} \sum_{p=1}^{2 d}\left[I I\left(e_{i}, e_{p}\right) \cdot I I\left(e_{j}, e_{p}\right) \cdot+g\left(I I\left(e_{i}, e_{p}\right), I I\left(e_{j}, e_{p}\right)\right)\right] \phi .
\end{aligned}
$$

Thus, we deduce

$$
\begin{aligned}
R^{E}(\phi)= & 2 \sum_{i, j=1}^{2 d} J\left(e_{j}\right) \cdot e_{j} \cdot \Omega_{N} \cdot \phi-\sum_{i, j, p=1}^{2 d} e_{i} \cdot e_{j} \cdot I I\left(e_{i}, e_{p}\right) \cdot I I\left(e_{j}, e_{p}\right) \cdot \phi \\
& -e_{i} \cdot e_{j} \cdot g\left(I I\left(e_{i}, e_{p}\right), I I\left(e_{j}, e_{p}\right)\right) \phi \\
= & -4 \Omega \cdot \Omega_{N} \cdot \phi-\sum_{i, j, p=1}^{2 d} e_{i} \cdot e_{j} \cdot I I\left(e_{i}, e_{p}\right) \cdot I I\left(e_{j}, e_{p}\right) \cdot \phi+|I I|^{2} \phi .
\end{aligned}
$$

Finally, the scalar product of the last equality with $\frac{\phi}{|\phi|^{2}}$ finishes the proof.

As we said in the proof of Corollary 3.2, the integer $r$ can be chosen such that $r \leq \frac{d-1}{2}$ if $d$ is odd and $r \leq \frac{d-2}{2}$ if $d$ is even. However, we note that a priori no such choice can be made for $s$ once $r$ has been fixed. In particular, one cannot conclude that the smallest twisted Dirac eigenvalue of a totally geodesic $M$ in $\widetilde{M}$ is $(d+1)^{2}$, even in the "simplest" case where $M=\mathbb{C P}^{d}$ (the $d$-dimensional complex projective space). To test the sharpness of the estimate (2.7), we compute in the following section the spectrum of $D_{M}^{\Sigma N}$ for $M=\mathbb{C P}^{d}$ canonically embedded in $\mathbb{C P}^{n}$.

\section{The spectrum of the twisted Dirac operator $D_{M}^{\Sigma N}$ on the com- plex projective space}

In this section, we compute the spectrum of the Dirac operator of $\mathbb{C P}^{d}$ twisted with the spinor bundle of its normal bundle when considered as canonically embedded in $\mathbb{C P}^{n}$. The eigenvalues will be deduced from M. Ben Halima's computations [3, Thm. 1]. We also need to compute the multiplicities in order to compare the upper bound in (2.7) with an eigenvalue which may be greater than the smallest one. The results are gathered in Theorems 4.7 and 4.8 below.

\subsection{The complex projective space as a symmetric space}

Consider the $d$-dimensional complex projective space $\mathbb{C P}^{d}$ as the right quotient $\mathrm{SU}_{d+1 /} / \mathrm{S}_{(}\left(\mathrm{U}_{d} \times \mathrm{U}_{1}\right)$, where $\mathrm{S}\left(\mathrm{U}_{d} \times \mathrm{U}_{1}\right):=\left\{\left(\begin{array}{ll}B & 0 \\ 0 & \operatorname{det}(B)^{-1}\end{array}\right) \mid B \in \mathrm{U}_{d}\right\}$. In this section we want to describe its tangent bundle and its normal bundle when canonically embedded into $\mathbb{C P}^{n}$ as homogeneous bundles, that is, as bundles associated to the $\mathrm{S}\left(\mathrm{U}_{d} \times \mathrm{U}_{1}\right)$-principal bundle $\mathrm{SU}_{d+1} \longrightarrow \mathbb{C P}^{d}$ via some linear representation of $\mathrm{S}\left(\mathrm{U}_{d} \times \mathrm{U}_{1}\right)$. The one corresponding to the tangent bundle is called the isotropy representation of the homogeneous space $\mathrm{SU}_{d+1 / \mathrm{S}}\left(\mathrm{U}_{d} \times \mathrm{U}_{1}\right)$. To compute it explicitly we consider the following $\operatorname{Ad}\left(\mathrm{S}\left(\mathrm{U}_{d} \times \mathrm{U}_{1}\right)\right)$-invariant 
complementary subspace

$$
\mathfrak{m}:=\left\{\left(\begin{array}{cccc}
0 & \ldots & 0 & z_{1} \\
\vdots & & \vdots & \vdots \\
0 & \ldots & 0 & z_{d} \\
-\overline{z_{1}} & \ldots & -\overline{z_{d}} & 0
\end{array}\right) \mid\left(z_{1}, \ldots, z_{d}\right) \in \mathbb{C}^{d}\right\}
$$

to the Lie-Algebra $\mathfrak{h}$ of $\mathrm{S}\left(\mathrm{U}_{d} \times \mathrm{U}_{1}\right)$ in the Lie-algebra $\mathfrak{s u}_{d+1}=\left\{X \in \mathbb{C}(d+1) \mid X^{*}=-X\right.$ and $\left.\operatorname{tr}(X)=0\right\}$ and fix the (real) basis $\left(A_{1}, J\left(A_{1}\right), \ldots, A_{d}, J\left(A_{d}\right)\right)$ of $\mathfrak{m}$, where:

- $\left(A_{l}\right)_{j k}=1$ if $(j, k)=(l, d+1),-1$ if $(j, k)=(d+1, l)$ and 0 otherwise;

- $\left(J\left(A_{l}\right)\right)_{j k}=i$ if $(j, k)=(l, d+1)$ or $(j, k)=(d+1, l)$ and 0 otherwise.

It is easy to check that $J$ defines a complex structure on $\mathfrak{m}$, which then makes $\mathfrak{m}$ into a $d$-dimensional complex vector space, and that $[\mathfrak{m}, \mathfrak{m}] \subset \mathfrak{h}$. In particular $\mathbb{C P}^{d}$ is a symmetric space.

Lemma 4.1 The isotropy representation of the symmetric space $\mathrm{SU}_{d+1 / \mathrm{S}}\left(\mathrm{U}_{d} \times \mathrm{U}_{1}\right)$ is given in the complex basis $\left(A_{1}, \ldots, A_{d}\right)$ of $\mathfrak{m}$ by:

$$
\begin{aligned}
\alpha: \mathrm{S}\left(\mathrm{U}_{d} \times \mathrm{U}_{1}\right) & \longrightarrow \mathrm{U}_{d} \\
\left(\begin{array}{cc}
B & 0 \\
0 & \operatorname{det}(B)^{-1}
\end{array}\right) & \longmapsto \operatorname{det}(B) \cdot B .
\end{aligned}
$$

Proof: For $k \in\{1, \ldots, d\}$ and $B \in \mathrm{U}_{d}$ we compute

$$
\begin{aligned}
& \operatorname{Ad}\left(\left(\begin{array}{cc}
B & 0 \\
0 & \operatorname{det}(B)^{-1}
\end{array}\right)\right)\left(A_{k}\right)=\left(\begin{array}{cc}
B & 0 \\
0 & \operatorname{det}(B)^{-1}
\end{array}\right) \cdot A_{k} \cdot\left(\begin{array}{cc}
B^{*} & 0 \\
0 & \operatorname{det}(B)
\end{array}\right) \\
& =\left(\begin{array}{cc}
B & 0 \\
0 & \operatorname{det}(B)^{-1}
\end{array}\right) \cdot\left(\begin{array}{cccc}
0 & \ldots & 0 & 0 \\
\vdots & & \vdots & 0 \\
0 & \ldots & 0 & \operatorname{det}(B) \\
\vdots & & \vdots & 0 \\
-B_{k 1}^{*} & \ldots & -B_{k d}^{*} & 0
\end{array}\right) \\
& =\left(\begin{array}{cccc}
0 & \cdots & 0 & \operatorname{det}(B) B_{1 k} \\
\vdots & & \vdots & \vdots \\
0 & \ldots & 0 & \operatorname{det}(B) B_{d k} \\
-\operatorname{det}(B)^{-1} B_{k 1}^{*} & \cdots & -\operatorname{det}(B)^{-1} B_{k d}^{*} & 0
\end{array}\right) \\
& =\sum_{j=1}^{d} \Re e\left(\operatorname{det}(B) B_{j k}\right) A_{j}+\Im m\left(\operatorname{det}(B) B_{j k}\right) J\left(A_{j}\right) \\
& =\sum_{j=1}^{d} \operatorname{det}(B) B_{j k} A_{j}
\end{aligned}
$$

which gives the result.

Recall that the tautological bundle of $\mathbb{C P}^{d}$ is the complex line bundle $\gamma_{d} \longrightarrow \mathbb{C P}^{d}$ defined by

$$
\gamma_{d}:=\left\{([z], v) \mid[z] \in \mathbb{C P}^{d} \text { and } v \in[z]\right\} .
$$

It carries a canonical Hermitian metric defined by $\left\langle([z], v),\left([z], v^{\prime}\right)\right\rangle:=\left\langle v, v^{\prime}\right\rangle$.

Lemma 4.2 The normal bundle $T^{\perp} \mathbb{C P}^{d}$ of the canonical embedding $\mathbb{C P}^{d} \rightarrow \mathbb{C P}^{n},[z] \mapsto\left[z, 0_{n-d}\right]$, is unitarily isomorphic to $\gamma_{d}^{*} \otimes \mathbb{C}^{n-d}$, where $\gamma_{d} \longrightarrow \mathbb{C P}^{d}$ is the tautological bundle of $\mathbb{C P}^{d}$ and $\mathbb{C}^{n-d}$ carries its canonical Hermitian inner product. In particular, the homogeneous bundle $T^{\perp} \mathbb{C P}^{d} \rightarrow \mathbb{C P}^{d}$ is associated to the $\mathrm{S}\left(\mathrm{U}_{d} \times \mathrm{U}_{1}\right)$-principal bundle $\mathrm{SU}_{d+1} \longrightarrow \mathbb{C P}^{d}$ via the representation

$$
\begin{aligned}
& \rho: \mathrm{S}\left(\mathrm{U}_{d} \times \mathrm{U}_{1}\right) \quad \longrightarrow \mathrm{U}_{n-d} \\
& \left(\begin{array}{cc}
B & 0 \\
0 & \operatorname{det}(B)^{-1}
\end{array}\right) \longmapsto \operatorname{det}(B) \mathrm{I}_{n-d} \text {. }
\end{aligned}
$$


Proof: Consider the map

$$
\begin{aligned}
\mathbb{C P}^{d} \times \mathbb{C}^{n-d} & \stackrel{\phi}{\longrightarrow} \gamma_{d} \otimes T^{\perp} \mathbb{C P}^{d} \\
([z], v) & \longmapsto([z], z) \otimes d_{z} \pi\left(0_{d+1}, v\right),
\end{aligned}
$$

where $\pi: \mathbb{C}^{n+1} \longrightarrow \mathbb{C P}^{n}$ is the canonical projection. It can be easily checked that $\phi$ is well-defined (the identity $\pi(\lambda z)=\pi(z)$ implies $\left.d_{z} \pi=\lambda d_{\lambda z} \pi\right)$ and is a unitary vector-bundle-isomorphism. This shows the first statement. Let $\left(e_{1}, \ldots, e_{d+1}\right)$ denote the canonical basis of $\mathbb{C}^{d+1}$. The map

$$
\begin{aligned}
\mathrm{SU}_{d+1} \times \mathbb{C} & \longrightarrow \gamma_{d} \\
(A, \lambda) & \longmapsto\left(\left[A e_{d+1}\right], \lambda A e_{d+1}\right)
\end{aligned}
$$

induces a complex vector-bundle-isomorphism $\mathrm{SU}_{d+1} \times \mathbb{C}_{\mathrm{S}}\left(\mathrm{U}_{d} \times \mathrm{U}_{1}\right) \longrightarrow \gamma_{d}$, where the right action of $\mathrm{S}\left(\mathrm{U}_{d} \times \mathrm{U}_{1}\right)$ onto $\mathrm{SU}_{d+1} \times \mathbb{C}$ is given by $(A, \lambda) \cdot\left(\begin{array}{cc}B & 0 \\ 0 & \operatorname{det}(B)^{-1}\end{array}\right):=\left(A \cdot\left(\begin{array}{cc}B & 0 \\ 0 & \operatorname{det}(B)^{-1}\end{array}\right), \operatorname{det}(B) \lambda\right)$. Thus $\gamma_{d}$ is isomorphic to the homogeneous bundle over $\mathbb{C P}^{d}$ which is associated to the $\mathrm{S}\left(\mathrm{U}_{d} \times \mathrm{U}_{1}\right)$-principal bundle $\mathrm{SU}_{d+1} \longrightarrow \mathbb{C P}^{d}$ via the representation $\mathrm{S}\left(\mathrm{U}_{\mathrm{d}} \times \mathrm{U}_{1}\right) \rightarrow \mathrm{U}_{1},\left(\begin{array}{cc}B & 0 \\ 0 & \operatorname{det}(B)^{-1}\end{array}\right) \mapsto \operatorname{det}(B)^{-1}$. This concludes the proof.

Note in particular that $T^{\perp} \mathbb{C P}^{d}$ is not trivial (and hence not flat because of $\pi_{1}\left(\mathbb{C P}^{d}\right)=0$ ).

\subsection{Spin structures on $T \mathbb{C P}^{d}$ and $T^{\perp} \mathbb{C P}^{d}$}

From now on we assume that both $d$ and $n$ are odd integers. Then both $T \mathbb{C P}^{d}$ and $T \mathbb{C P}^{n}$ are spin, in particular $T^{\perp} \mathbb{C P}^{d}$ is spin. Since $\mathbb{C P}^{d}$ is simply-connected, there is a unique spin structure on $T \mathbb{C P}^{d}$ and on $T^{\perp} \mathbb{C P}^{d}$. In this section we describe those spin structures as homogeneous spin structures. For that purpose one looks for Lie-group-homomorphisms $\mathrm{S}\left(\mathrm{U}_{d} \times \mathrm{U}_{1}\right) \stackrel{\tilde{\alpha}}{\rightarrow} \operatorname{Spin}_{2 d}$ and $\mathrm{S}\left(\mathrm{U}_{d} \times \mathrm{U}_{1}\right) \stackrel{\tilde{\rho}}{\rightarrow} \operatorname{Spin}_{2(n-d)}$ lifting $\alpha$ and $\rho$ through the non-trivial two-fold-covering map $\operatorname{Spin}_{2 k} \stackrel{\xi}{\longrightarrow} \mathrm{SO}_{2 k}$.

First we recall the existence for any positive integer $k$ of a Lie-group homomorphism $\mathrm{U}_{k} \stackrel{j}{\longrightarrow} \operatorname{Spin}_{2 k}^{c}$ with $\xi^{c} \circ j=\iota$, where $\operatorname{Spin}_{2 k}^{c}:=\operatorname{Spin}_{2 k} \times \mathrm{U}_{1} \mathbb{Z}_{2}$ is the $\operatorname{spin}^{c}$ group, $\xi^{c}: \operatorname{Spin}_{2 k}^{c} \longrightarrow \mathrm{SO}_{2 k} \times \mathrm{U}_{1},[u, z] \mapsto\left(\xi(u), z^{2}\right)$ is the canonical two-fold-covering map and $\iota: \mathrm{U}_{k} \longrightarrow \mathrm{SO}_{2 k} \times \mathrm{U}_{1}, A \mapsto\left(A_{\mathbb{R}}, \operatorname{det}(A)\right)$. The Lie-group homomorphism $j$ can be explicitly described on elements of $\mathrm{U}_{k}$ of diagonal form as:

$$
j\left(\operatorname{diag}\left(e^{i \lambda_{1}}, \ldots, e^{i \lambda_{k}}\right)\right)=e^{\frac{i}{2}\left(\sum_{j=1}^{k} \lambda_{j}\right)} \cdot \widetilde{R}_{e_{1}, J\left(e_{1}\right)}\left(\frac{\lambda_{1}}{2}\right) \cdot \ldots \cdot \widetilde{R}_{e_{k}, J\left(e_{k}\right)}\left(\frac{\lambda_{k}}{2}\right),
$$

where $J$ is the canonical complex structure on $\mathbb{C}^{k}$ and, for any orthonormal system $\{v, w\}$ in $\mathbb{R}^{2 k}$ and $\lambda \in \mathbb{R}$, the element $\widetilde{R}_{v, w}(\lambda) \in \operatorname{Spin}_{2 k}$ is defined by

$$
\widetilde{R}_{v, w}(\lambda):=\cos (\lambda)+\sin (\lambda) v \cdot w .
$$

To keep the notations simple we denote by $j$ both such Lie-group-homomorphisms $\mathrm{U}_{d} \longrightarrow \operatorname{Spin}_{2 d}^{c}$ and $\mathrm{U}_{n-d} \longrightarrow \operatorname{Spin}_{2(n-d)}^{c}$.

Lemma 4.3 Let $d<n$ be odd integers.

1. The spin structure on $T \mathbb{C P}^{d}$ is associated to the $\mathrm{S}\left(\mathrm{U}_{d} \times \mathrm{U}_{1}\right)$-principal bundle $\mathrm{SU}_{d+1} \longrightarrow \mathbb{C P}^{d}$ via the Lie-group-homomorphism

$$
\begin{aligned}
\tilde{\alpha}: \mathrm{S}\left(\mathrm{U}_{d} \times \mathrm{U}_{1}\right) & \longrightarrow \operatorname{Spin}_{2 d} \\
\left(\begin{array}{cc}
B & 0 \\
0 & \operatorname{det}(B)^{-1}
\end{array}\right) & \longmapsto \operatorname{det}(B)^{-\frac{d+1}{2}} \cdot j \circ \alpha\left(\left(\begin{array}{cc}
B & 0 \\
0 & \operatorname{det}(B)^{-1}
\end{array}\right)\right) .
\end{aligned}
$$

2. The spin structure on $T^{\perp} \mathbb{C P}^{d}$ is associated to the $\mathrm{S}\left(\mathrm{U}_{d} \times \mathrm{U}_{1}\right)$-principal bundle $\mathrm{SU}_{d+1} \longrightarrow \mathbb{C P}^{d}$ via the Lie-group-homomorphism

$$
\begin{aligned}
& \tilde{\rho}: \mathrm{S}\left(\mathrm{U}_{d} \times \mathrm{U}_{1}\right) \quad \longrightarrow \operatorname{Spin}_{2(n-d)} \\
& \left(\begin{array}{cc}
B & 0 \\
0 & \operatorname{det}(B)^{-1}
\end{array}\right) \longmapsto \operatorname{det}(B)^{-\frac{n-d}{2}} \cdot j \circ \rho\left(\left(\begin{array}{cc}
B & 0 \\
0 & \operatorname{det}(B)^{-1}
\end{array}\right)\right) \text {. }
\end{aligned}
$$


Proof: It suffices to prove the results for elements of $\mathrm{S}\left(\mathrm{U}_{d} \times \mathrm{U}_{1}\right)$ of diagonal form. Indeed any element of $\mathrm{S}\left(\mathrm{U}_{d} \times \mathrm{U}_{1}\right)$ is conjugated in $\mathrm{SU}_{d+1}$ to such a diagonal matrix. Since $\mathrm{SU}_{d+1}$ is simply-connected the map $\mathrm{SU}_{d+1} \rightarrow \mathrm{SO}_{2 k} \times \mathrm{U}_{1}, P \mapsto\left(P A P^{-1}, \operatorname{det}(A)\right.$ ) (where $A \in U_{k}$ is arbitrary), admits a lift through $\operatorname{Spin}_{2 k}^{c} \stackrel{\xi^{c}}{\longrightarrow} \mathrm{SO}_{2 k} \times \mathrm{U}_{1}$ which is uniquely determined by the image of one single point. Therefore the lifts under consideration are uniquely determined on diagonal elements.

For $\theta_{1}, \ldots, \theta_{d} \in \mathbb{R}$ let $M_{\theta_{1}, \ldots, \theta_{d}}:=\operatorname{diag}\left(e^{i \theta_{1}}, \ldots, e^{i \theta_{d}}, e^{-i\left(\sum_{j=1}^{d} \theta_{j}\right)}\right) \in \mathrm{S}\left(\mathrm{U}_{d} \times \mathrm{U}_{1}\right)$. Then

$$
u_{\theta_{1}, \ldots, \theta_{d}}:=\widetilde{R}_{e_{1}, J\left(e_{1}\right)}\left(\frac{\theta_{1}+\sum_{j=1}^{d} \theta_{j}}{2}\right) \cdot \ldots \cdot \widetilde{R}_{e_{d}, J\left(e_{d}\right)}\left(\frac{\theta_{d}+\sum_{j=1}^{d} \theta_{j}}{2}\right)
$$

lies in $\operatorname{Spin}_{2 d}$, only depends on $\left[\theta_{1}, \ldots, \theta_{d}\right] \in \mathbb{R}^{d} / 2 \pi \mathbb{Z}^{d}$ (if some $\theta_{k}$ is replaced by $\theta_{k}+2 m \pi$, then $u_{\theta_{1}, \ldots, \theta_{d}}$ is replaced by $(-1)^{m(d-1)} u_{\theta_{1}, \ldots, \theta_{d}}$, and $d-1$ is even $)$ with $\xi\left(u_{\theta_{1}, \ldots, \theta_{d}}\right)=\alpha\left(M_{\theta_{1}, \ldots, \theta_{d}}\right)$. Therefore $\tilde{\alpha}\left(M_{\theta_{1}, \ldots, \theta_{d}}\right)=u_{\theta_{1}, \ldots, \theta_{d}}$. Moreover,

$$
\begin{aligned}
j \circ \alpha\left(M_{\theta_{1}, \ldots, \theta_{d}}\right) & =e^{\frac{i}{2}\left(\sum_{j=1}^{d} \theta_{j}+\sum_{k=1}^{d} \theta_{k}\right)} \cdot \widetilde{R}_{e_{1}, J\left(e_{1}\right)}\left(\frac{\theta_{1}+\sum_{j=1}^{d} \theta_{j}}{2}\right) \cdot \ldots \cdot \widetilde{R}_{e_{d}, J\left(e_{d}\right)}\left(\frac{\theta_{d}+\sum_{j=1}^{d} \theta_{j}}{2}\right) \\
& =e^{\frac{i(d+1)}{2} \sum_{j=1}^{d} \theta_{j}} \cdot \tilde{\alpha}\left(M_{\theta_{1}, \ldots, \theta_{d}}\right) \\
& =\operatorname{det}\left(\operatorname{diag}\left(e^{i \theta_{1}}, \ldots, e^{i \theta_{d}}\right)\right)^{\frac{d+1}{2}} \cdot \tilde{\alpha}\left(M_{\theta_{1}, \ldots, \theta_{d}}\right),
\end{aligned}
$$

which proves 1.

The other case is much the same: setting

$$
\tilde{\rho}\left(M_{\theta_{1}, \ldots, \theta_{d}}\right):=\widetilde{R}_{e_{1}, J\left(e_{1}\right)}\left(\frac{\sum_{j=1}^{d} \theta_{j}}{2}\right) \cdot \ldots \cdot \widetilde{R}_{e_{n-d}, J\left(e_{n-d}\right)}\left(\frac{\sum_{j=1}^{d} \theta_{j}}{2}\right),
$$

one obtains a well-defined Lie-group-homomorphism $\mathrm{S}\left(\mathrm{U}_{d} \times \mathrm{U}_{1}\right) \stackrel{\tilde{\rho}}{\rightarrow} \operatorname{Spin}_{2(n-d)}$ with $\xi \circ \tilde{\rho}=\rho$ (the integer $n-d$ is even) and

$$
\begin{aligned}
j \circ \rho\left(M_{\theta_{1}, \ldots, \theta_{d}}\right) & =e^{\frac{i}{2} \sum_{j=1}^{n-d} \sum_{k=1}^{d} \theta_{k}} \cdot \widetilde{R}_{e_{1}, J\left(e_{1}\right)}\left(\frac{\sum_{j=1}^{d} \theta_{j}}{2}\right) \cdot \ldots \cdot \widetilde{R}_{e_{n-d}, J\left(e_{n-d}\right)}\left(\frac{\sum_{j=1}^{d} \theta_{j}}{2}\right) \\
& =\operatorname{det}\left(\operatorname{diag}\left(e^{i \theta_{1}}, \ldots, e^{i \theta_{d}}\right)\right)^{\frac{n-d}{2}} \tilde{\rho}\left(M_{\theta_{1}, \ldots, \theta_{d}}\right),
\end{aligned}
$$

which shows 2 and concludes the proof.

In particular, we obtain the following

Corollary 4.4 Let $d<n$ be odd integers and consider the canonical embedding $\mathbb{C P}^{d} \rightarrow \mathbb{C P}^{n}$ as above. Then there exists a unitary and parallel isomorphism

$$
\Sigma\left(T^{\perp} \mathbb{C P}^{d}\right) \cong \bigoplus_{s=0}^{n-d}\left(\begin{array}{c}
n-d \\
s
\end{array}\right) \cdot \gamma_{d}^{\frac{n-d}{2}-s},
$$

where $\Sigma\left(T^{\perp} \mathbb{C P}^{d}\right)$ denotes the (complex) spinor bundle of $T^{\perp} \mathbb{C P}^{d}$ and, for each $s \in\{0, \ldots, n-d\}$, the factor $\left(\begin{array}{c}n-d \\ s\end{array}\right)$ stands for the multiplicity with which the line bundle $\gamma_{d}^{\frac{n-d}{2}-s}$ appears in the splitting.

Proof: By Lemma 4.3 and Lemma 4.2 one has, for any $B \in \mathrm{U}_{d}$ :

$$
\begin{aligned}
\tilde{\rho}\left(\left(\begin{array}{cc}
B & 0 \\
0 & \operatorname{det}(B)^{-1}
\end{array}\right)\right) & =\operatorname{det}(B)^{-\frac{n-d}{2}} \cdot j \circ \rho\left(\left(\begin{array}{cc}
B & 0 \\
0 & \operatorname{det}(B)^{-1}
\end{array}\right)\right) \\
& =\operatorname{det}(B)^{-\frac{n-d}{2}} \cdot j\left(\operatorname{det}(B) \mathrm{I}_{n-d}\right) .
\end{aligned}
$$

Now it is elementary to prove that, for any positive integer $k$, any $z \in \mathrm{U}_{1}$ and any $s \in\{0, \ldots, k\}$,

$$
\delta_{2 k} \circ j\left(z \cdot \mathrm{I}_{k}\right)_{\left.\right|_{2 k} ^{(s)}}=z^{s} \cdot \operatorname{Id}_{\Sigma_{2 k}^{(s)}},
$$


where $\Sigma_{2 k}^{(s)}$ is the eigenspace of the Clifford action of the Kähler form to the eigenvalue $i(2 s-k)$ in the spinor space $\Sigma_{2 k}$. In particular $\Sigma_{2 k}^{(s)}$ splits into the direct sum of $\operatorname{dim}_{\mathbb{C}}\left(\Sigma_{2 k}^{(s)}\right)$ copies of some onedimensional representation, with $\operatorname{dim}_{\mathbb{C}}\left(\Sigma_{2 k}^{(s)}\right)=\left(\begin{array}{c}k \\ s\end{array}\right)$. Since $\Sigma_{2 k}=\oplus_{s=0}^{k} \Sigma_{2 k}^{(s)}$, we obtain the following splitting:

$$
\begin{aligned}
\delta_{2(n-d)} \circ \tilde{\rho} & =\bigoplus_{s=0}^{n-d} \operatorname{det}(\cdot)^{-\left(\frac{n-d}{2}-s\right)} \otimes \operatorname{Id}_{\Sigma_{2(n-d)}^{(s)}} \\
& =\bigoplus_{s=0}^{n-d} \operatorname{det}(\cdot)^{-\left(\frac{n-d}{2}-s\right)} \otimes \mathbf{1}_{\mathbb{C}}\left(\begin{array}{c}
n-d \\
s
\end{array}\right),
\end{aligned}
$$

where $\operatorname{det}(\cdot): \mathrm{S}\left(\mathrm{U}_{d} \times \mathrm{U}_{1}\right) \rightarrow \mathrm{U}_{1},\left(\begin{array}{cc}B & 0 \\ 0 & \operatorname{det}(B)^{-1}\end{array}\right) \mapsto \operatorname{det}(B)$, the trivial representation on $\mathbb{C}$ is denoted by $\mathbf{1}_{\mathbb{C}}$ and " $\mathbf{1}_{\mathbb{C}}^{l}$ " means that this representation appears with multiplicity $l$.

\subsection{The twisted Dirac operator on $\mathbb{C P}^{d}$}

As a consequence of Corollary 4.4 the tensor product $\Sigma\left(T \mathbb{C P}^{d}\right) \otimes \Sigma\left(T^{\perp} \mathbb{C P}^{d}\right)$ splits into subbundles of the form $\Sigma\left(T \mathbb{C P}^{d}\right) \otimes \gamma_{d}^{m}$ for some integer $m$. Since this splitting is orthogonal and parallel, it is also preserved by the corresponding twisted Dirac operator. Hence it suffices to describe the Dirac operator of the twisted spinor bundle $\Sigma\left(T \mathbb{C P}^{d}\right) \otimes \gamma_{d}^{m}$ over $\mathbb{C P}^{d}$ as an infinite sum of matrices, where $m \in \mathbb{Z}$ is an arbitrary (non-necessarily positive) integer. The Dirac eigenvalues of $\Sigma\left(T \mathbb{C P}^{d}\right) \otimes \gamma_{d}^{m}$ have been computed by M. Ben Halima in [3, Thm. 1]. Indeed, we have

Theorem 4.5 For an odd integer d let $\mathbb{C P}^{d}$ be endowed with its Fubini-Study metric of constant holomorphic sectional curvature 4 . For an arbitrary $m \in \mathbb{Z}$ let the $m^{\text {th }}$ power $\gamma_{d}^{m}$ of the tautological bundle of $\mathbb{C P}^{d}$ be endowed with its canonical metric and connection. Then the eigenvalues (without multiplicities) of the square of the Dirac operator of $\mathbb{C P}^{d}$ twisted by $\gamma_{d}^{m}$ are given by the following families:

1. $2(r+l) \cdot(d+1+2(l-m-\epsilon))$, where $r \in\{1, \ldots, d-1\}, \epsilon \in\{0,1\}$ and $l \in \mathbb{N}$ with $l \geq \max \left(\epsilon, \frac{d+1}{2}-r+m\right)$.

2. $2 l(2 l+d-1-2 m)$, where $l \in \mathbb{N}, l \geq \max \left(0, m+\frac{d+1}{2}\right)$.

3. $2(d+l)(d+1+2(l-m))$, where $l \in \mathbb{N}, l \geq \max \left(0, m-\frac{d+1}{2}\right)$.

The first family of eigenvalues corresponds to an irreducible representation of $\mathrm{SU}_{d+1}$ with highest weight given by [3, Prop. 2]

$$
(r+2 l-\frac{d-1}{2}-m-\epsilon, \underbrace{r+l-\frac{d-1}{2}-m, \ldots, r+l-\frac{d-1}{2}-m}_{r-1}, r+l-\frac{d+1}{2}-m+\epsilon, \underbrace{r+l-\frac{d+1}{2}-m, \ldots, r+l}_{d-r-1}-\frac{d+1}{2}-m) .
$$

Similarly, the second family of eigenvalues corresponds to the highest weight

$$
(2 l-\frac{d+1}{2}-m, \underbrace{l-\frac{d+1}{2}-m, \ldots, l-\frac{d+1}{2}-m}_{d-1}) .
$$

The last family of eigenvalues corresponds to

$$
(2 l+\frac{d+1}{2}-m, \underbrace{\left.l+\frac{d+1}{2}-m, \ldots, l+\frac{d+1}{2}-m\right)}_{d-1} .
$$

In the following, we will determine the multiplicities of the eigenvalues in Theorem 4.5. Indeed, we have

Lemma 4.6 Let $d \geq 1$ be an odd integer and $m \in \mathbb{Z}$. 
1. The multiplicities of the first family of the eigenvalues are equal to

$$
\frac{d\left(\frac{d+1}{2}+r-m+2 l-\epsilon\right)}{(r+l)\left(\frac{d+1}{2}-m+l-\epsilon\right)} \cdot\left(\begin{array}{c}
d+l-\epsilon \\
d
\end{array}\right) \cdot\left(\begin{array}{c}
d-1 \\
d-r-\epsilon
\end{array}\right) \cdot\left(\begin{array}{c}
\frac{d-1}{2}+r-m+l \\
d
\end{array}\right) .
$$

2. For the second family, we have

$$
\prod_{k=2}^{d}\left(1+\frac{l}{k-1}\right) \cdot\left(1+\frac{2 l-\frac{d+1}{2}-m}{d}\right) \cdot \prod_{j=2}^{d}\left(1+\frac{l-\frac{d+1}{2}-m}{d-j+1}\right) .
$$

3. For the last family of eigenvalues, the multiplicities are equal to

$$
\prod_{k=2}^{d}\left(1+\frac{l}{k-1}\right) \cdot\left(1+\frac{2 l+\frac{d+1}{2}-m}{d}\right) \cdot \prod_{j=2}^{d}\left(1+\frac{l+\frac{d+1}{2}-m}{d-j+1}\right) .
$$

In our convention, a product taken on an empty index-set is equal to 1.

Proof: The required multiplicity can be computed with the help of the Weyl's character formula [2]

$$
\prod_{\alpha \in \Delta_{+}}\left(1+\frac{\langle\lambda, \alpha\rangle}{\left\langle\delta_{+}, \alpha\right\rangle}\right)
$$

where $\lambda$ is a highest weight of an irreducible $\mathrm{SU}_{d+1}$-representation and $\Delta_{+}$is the set of positive roots, i.e.

$$
\Delta_{+}=\left\{\theta_{j}-\theta_{k}, 1 \leq j<k \leq d, \theta_{j}+\sum_{k=1}^{d} \theta_{k}, 1 \leq j \leq d\right\}
$$

and $\delta_{+}=\sum_{k=1}^{d}(d-k+1) \theta_{k}$ is the half-sum of the positive roots of $\mathrm{SU}_{d+1}$, see [3, p. 442]. Here the scalar product $\langle.,$.$\rangle is the Riemannian metric on the dual of a maximal torus of \mathrm{SU}_{d+1}$, which is defined by the following product of matrices $\left\langle\lambda, \lambda^{\prime}\right\rangle=\lambda \cdot \beta \cdot{ }^{t} \lambda^{\prime}$ where $\beta$ is the matrix given by $\frac{2}{d+1}\left(-1+(d+1) \delta_{j k}\right)_{1 \leq j, k \leq d}$. To compute the quotient in the Weyl's character formula, we treat the three cases separately:

1. Consider $\alpha$ of the form $\alpha=\theta_{j}-\theta_{k}$ for some $1 \leq j<k \leq d$. Note that this form for $\alpha$ can only exist if $d>1$. We compute

$$
\beta \cdot \alpha=\beta \cdot\left(\begin{array}{c}
0 \\
\vdots \\
0 \\
1 \\
0 \\
\vdots \\
0 \\
-1 \\
0 \\
\vdots \\
0
\end{array}\right)
$$


$=\frac{2}{d+1}\left(\begin{array}{cccc}d & -1 & \ldots & -1 \\ -1 & \ddots & & \vdots \\ \vdots & & \ddots & -1 \\ -1 & \ldots & -1 & d\end{array}\right) \cdot\left(\begin{array}{c}0 \\ \vdots \\ 0 \\ 1 \\ 0 \\ \vdots \\ 0 \\ -1 \\ 0 \\ \vdots \\ 0\end{array}\right)$

$=\frac{2}{d+1}\left(\begin{array}{c}0 \\ \vdots \\ 0 \\ d+1 \\ 0 \\ \vdots \\ 0 \\ -d-1 \\ 0 \\ \vdots \\ 0\end{array}\right)$

Therefore,

$$
\begin{aligned}
\left\langle\delta_{+}, \alpha\right\rangle=2(d, d-1, \ldots, 1) \cdot\left(\begin{array}{c}
0 \\
\vdots \\
0 \\
1 \\
0 \\
\vdots \\
0 \\
-1 \\
0 \\
\vdots \\
0
\end{array}\right) \\
=2(d-j+1-(d-k+1)) \\
=2(k-j) .
\end{aligned}
$$

For the highest weight $\lambda$ corresponding to the first family of eigenvalues, we denote by $u^{\prime}$ the first component, $\vec{u}_{+}$the $r-1$ components, $u$ the $r$-components and by $\vec{u}_{-}$the last $d-r-1$ components. 
Thus, we have

$$
\begin{aligned}
& \langle\lambda, \alpha\rangle=2\left(u^{\prime}, \vec{u}_{+}, u, \vec{u}_{-}\right) \cdot\left(\begin{array}{c}
0 \\
\vdots \\
0 \\
1 \\
0 \\
\vdots \\
0 \\
-1 \\
0 \\
\vdots \\
0
\end{array}\right) \\
& =\mid \begin{array}{lll}
2\left(u^{\prime}-u_{+}\right) & \text {case } j=1, k \in\{2, \ldots, r\} \\
2\left(u^{\prime}-u\right) & \text { case } j=1, k=r+1 \\
2\left(u^{\prime}-u_{-}\right) & \text {case } j=1, k \in\{r+2, \ldots, d\} \\
0 & \text { case } j, k \in\{2, \ldots, r\} \\
2\left(u_{+}-u\right) & \text { case } j \in\{2, \ldots, r\}, k=r+1 \\
2\left(u_{+}-u_{-}\right) & \text {case } j \in\{2, \ldots, r\}, k \in\{r+2, \ldots, d\} \\
2\left(u-u_{-}\right) & \text {case } j=r+1, k \in\{r+2, \ldots, d\} \\
0 & \text { case } j, k \in\{r+2, \ldots, d\}
\end{array} \\
& =\mid \begin{array}{lll}
2(l-\epsilon) & \text { case } j=1, k \in\{2, \ldots, r\} \\
2(l+1-2 \epsilon) & \text { case } j=1, k=r+1 \\
2(l+1-\epsilon) & \text { case } j=1, k \in\{r+2, \ldots, d\} \\
0 & \text { case } j, k \in\{2, \ldots, r\} \\
2(1-\epsilon) & \text { case } j \in\{2, \ldots, r\}, k=r+1 \\
2 & \text { case } j \in\{2, \ldots, r\}, k \in\{r+2, \ldots, d\} \\
2 \epsilon & \text { case } j=r+1, k \in\{r+2, \ldots, d\} \\
0 & \text { case } j, k \in\{r+2, \ldots, d\} .
\end{array}
\end{aligned}
$$

We obtain, for $\alpha=\theta_{j}-\theta_{k}$ with $1 \leq j<k \leq d$ :

$$
1+\frac{\langle\lambda, \alpha\rangle}{\left\langle\delta_{+}, \alpha\right\rangle}=\mid \begin{array}{lll}
\frac{l-\epsilon+k-j}{k-j} & \text { case } j=1, k \in\{2, \ldots, r\} \\
\frac{l+1-2 \epsilon+k-j}{k-j} & \text { case } j=1, k=r+1 \\
\frac{l+1-\epsilon+k-j}{k-j} & \text { case } j=1, k \in\{r+2, \ldots, d\} \\
1 & \text { case } j, k \in\{2, \ldots, r\} \\
\frac{1-\epsilon+k-j}{k-j} & \text { case } j \in\{2, \ldots, r\}, k=r+1 \\
\frac{1+k-j}{k-j} & \text { case } j \in\{2, \ldots, r\}, k \in\{r+2, \ldots, d\} \\
\frac{\epsilon+k-j}{k-j} & \text { case } j=r+1, k \in\{r+2, \ldots, d\} \\
1 & \text { case } j, k \in\{r+2, \ldots, d\} .
\end{array}
$$

Now choose $\alpha=\theta_{j}+\sum_{k=1}^{d} \theta_{k}$ with $j \in\{1, \ldots, d\}$, then

$$
\beta \cdot \alpha=\beta \cdot\left(\begin{array}{c}
1 \\
\vdots \\
1 \\
2 \\
1 \\
\vdots \\
1
\end{array}\right)
$$




$$
\begin{aligned}
& =\frac{2}{d+1}\left(\begin{array}{cccc}
d & -1 & \ldots & -1 \\
-1 & \ddots & & \vdots \\
\vdots & & \ddots & -1 \\
-1 & \ldots & -1 & d
\end{array}\right) \cdot\left(\begin{array}{c}
1 \\
\vdots \\
1 \\
2 \\
1 \\
\vdots \\
1
\end{array}\right) \\
& =\frac{2}{d+1}\left(\begin{array}{c}
0 \\
\vdots \\
0 \\
2 d-(d-1) \\
0 \\
\vdots \\
0
\end{array}\right) \\
& =2 \theta_{j} .
\end{aligned}
$$

Therefore,

$$
\begin{aligned}
\left\langle\delta_{+}, \alpha\right\rangle & =2(d, d-1, \ldots, 1) \cdot\left(\begin{array}{c}
0 \\
\vdots \\
0 \\
1 \\
0 \\
\vdots \\
0
\end{array}\right) \\
& =2(d-j+1) .
\end{aligned}
$$

Using the same notations as above, we compute

$$
\begin{aligned}
\langle\lambda, \alpha\rangle=2\left(u^{\prime}, \vec{u}_{+}, u, \vec{u}_{-}\right) \cdot\left(\begin{array}{c}
0 \\
\vdots \\
0 \\
1 \\
0 \\
\vdots \\
0
\end{array}\right) \\
= \\
=\quad \begin{array}{lll}
2 u^{\prime} & \text { case } j=1 \\
2 u_{+} & \text {case } & j \in\{2, \ldots, r\} \\
2 u & \text { case } j=r+1 \\
2 u_{-} & \text {case } & j \in\{r+2, \ldots, d\} \\
2\left(u_{-}+1+l-\epsilon\right) & \text { case } j=1 \\
2\left(u_{-}+1\right) & \text { case } j \in\{2, \ldots, r\} \\
2\left(u_{-}+\epsilon\right) & \text { case } j=r+1 \\
2 u_{-} & \text {case } j \in\{r+2, \ldots, d\} .
\end{array}
\end{aligned}
$$

We obtain, for $\alpha=\theta_{j}+\sum_{k=1}^{d} \theta_{k}$ with $j \in\{1, \ldots, d\}$ :

$$
1+\frac{\langle\lambda, \alpha\rangle}{\left\langle\delta_{+}, \alpha\right\rangle}=\mid \begin{array}{lll}
\frac{u_{-}+1+l-\epsilon+d-j+1}{d-j+1} & \text { case } j=1 \\
\frac{u_{-}+1+d-j+1}{d-j+1} & \text { case } j \in\{2, \ldots, r\} \\
\frac{u_{-}+\epsilon+d-j+1}{d-j+1} & \text { case } j=r+1 \\
\frac{u_{-}+d-j+1}{d-j+1} & \text { case } j \in\{r+2, \ldots, d\} .
\end{array}
$$


In order to compute the product we separate both cases $\epsilon=0$ and $\epsilon=1$.

- Case $\epsilon=0$ : Then

$$
\begin{aligned}
& \prod_{\alpha \in \Delta_{+}}\left(1+\frac{\langle\lambda, \alpha\rangle}{\left\langle\delta_{+}, \alpha\right\rangle}\right)=\left(\prod_{k=2}^{r} \frac{l+k-1}{k-1}\right) \cdot\left(\prod_{k=r+1}^{d} \frac{l+k}{k-1}\right) \cdot\left(\prod_{j=2}^{r} \prod_{k=r+1}^{d} \frac{k+1-j}{k-j}\right) \cdot \\
& \frac{u_{-}+l+d+1}{d} \cdot\left(\prod_{j=2}^{r} \frac{u_{-}+d-j+2}{d-j+1}\right) \cdot\left(\prod_{j=r+1}^{d} \frac{u_{-}+d-j+1}{d-j+1}\right) \\
&=\frac{(l+1) \cdot \ldots \cdot(l+r-1) \cdot(l+r+1) \cdot \ldots \cdot(l+d)}{1 \cdot 2 \cdot \ldots \cdot(d-1)} \cdot \\
& \quad\left(\prod_{j=2}^{r} \frac{(r+2-j) \cdot \ldots \cdot(d+1-j)}{(r+1-j) \cdot \ldots \cdot(d-j)}\right) \cdot \frac{u_{-}+l+d+1}{d} \cdot \\
& \quad \frac{\left(u_{-}+d\right) \cdot \ldots \cdot\left(u_{-}+d-r+2\right)}{(d-1) \cdot \ldots \cdot(d-r+1)} \cdot \frac{\left(u_{-}+d-r\right) \cdot \ldots \cdot\left(u_{-}+1\right)}{(d-r) \cdot \ldots \cdot 2 \cdot 1} \\
&=\frac{d}{l+r} \cdot \frac{(l+d) !}{d ! \cdot l !} \cdot\left(\prod_{j=2}^{r} \frac{d+1-j}{r+1-j}\right) \cdot \frac{u_{-}+l+d+1}{u_{-}+d-r+1} \cdot \frac{\left(u_{-}+d\right) !}{d ! \cdot u_{-} !} \\
&=\frac{d\left(\begin{array}{c}
l+d \\
d
\end{array}\right) \cdot \frac{(d-1) \cdot \ldots \cdot(d+1-r)}{(r-1) \cdot \ldots \cdot 2 \cdot 1} \cdot \frac{u_{-}+l+d+1}{u_{-}+d-r+1} \cdot\left(\begin{array}{c}
u_{-}+d \\
d
\end{array}\right)}{(l+r)\left(u_{-}+d-r+d\right)} \\
&=
\end{aligned}
$$

which gives for the multiplicity in this case (replace $u_{-}$by $r-\frac{d+1}{2}-m+l$ ):

$$
\prod_{\alpha \in \Delta_{+}}\left(1+\frac{\langle\lambda, \alpha\rangle}{\left\langle\delta_{+}, \alpha\right\rangle}\right)=\frac{d\left(\frac{d+1}{2}+r-m+2 l\right)}{(r+l)\left(\frac{d+1}{2}-m+l\right)} \cdot\left(\begin{array}{c}
d+l \\
d
\end{array}\right) \cdot\left(\begin{array}{c}
d-1 \\
d-r
\end{array}\right) \cdot\left(\begin{array}{c}
\frac{d-1}{2}+r-m+l \\
d
\end{array}\right) .
$$

- Case $\epsilon=1$ : Then

$$
\begin{aligned}
& \prod_{\alpha \in \Delta_{+}}\left(1+\frac{\langle\lambda, \alpha\rangle}{\left\langle\delta_{+}, \alpha\right\rangle}\right)=\left(\prod_{k=2}^{r+1} \frac{l+k-2}{k-1}\right) \cdot\left(\prod_{k=r+2}^{d} \frac{l+k-1}{k-1}\right) \cdot\left(\prod_{j=2}^{r+1} \prod_{k=r+2}^{d} \frac{k+1-j}{k-j}\right) \cdot \\
& \frac{u_{-}+l+d}{d} \cdot\left(\prod_{j=2}^{r+1} \frac{u_{-}+d-j+2}{d-j+1}\right) \cdot\left(\prod_{j=r+2}^{d} \frac{u_{-}+d-j+1}{d-j+1}\right) \\
&=\frac{l \cdot \ldots \cdot(l+r-1) \cdot(l+r+1) \cdot \ldots \cdot(l+d-1)}{1 \cdot 2 \cdot \ldots \cdot(d-1)} \cdot \\
& \quad\left(\prod_{j=2}^{r+1} \frac{(r+3-j) \cdot \ldots \cdot(d+1-j)}{(r+2-j) \cdot \ldots \cdot(d-j)}\right) \cdot \frac{u_{-}+l+d}{d} \cdot \\
& \frac{\left(u_{-}+d\right) \cdot \ldots \cdot\left(u_{-}+d-r+1\right)}{(d-1) \cdot \ldots \cdot(d-r)} \cdot \frac{(u-+d-r-1) \cdot \ldots \cdot\left(u_{-}+1\right)}{(d-r-1) \cdot \ldots \cdot 2 \cdot 1} \\
&=\frac{d}{l+r} \cdot \frac{(l+d-1) !}{d ! \cdot(l-1) !} \cdot\left(\prod_{j=2}^{r+1} \frac{d+1-j}{r+2-j}\right) \cdot \frac{u_{-}+l+d}{u_{-}+d-r} \cdot \frac{\left(u_{-}+d\right) !}{u_{-} ! \cdot d !} \\
&=\frac{d\left(u_{-}+l+d\right)}{(l+r)\left(u_{-}+d-r\right)} \cdot \frac{(l+d-1) !}{d ! \cdot(l-1) !} \cdot \frac{(d-1) !}{r ! \cdot(d-r-1) !} \cdot \frac{\left(u_{-}+d\right) !}{u_{-} ! \cdot d !} \\
&(l+r)\left(u_{-}+l+d\right) \\
&=\left(\begin{array}{c}
l+d-1 \\
d
\end{array}\right) \cdot\left(\begin{array}{c}
d-1 \\
r
\end{array}\right) \cdot\left(\begin{array}{c}
u_{-}+d \\
d
\end{array}\right),
\end{aligned}
$$


which, replacing $u_{-}$by $r-\frac{d+1}{2}-m+l$, gives

$$
\prod_{\alpha \in \Delta_{+}}\left(1+\frac{\langle\lambda, \alpha\rangle}{\left\langle\delta_{+}, \alpha\right\rangle}\right)=\frac{d\left(\frac{d-1}{2}+r-m+2 l\right)}{(r+l)\left(\frac{d-1}{2}-m+l\right)} \cdot\left(\begin{array}{c}
d+l-1 \\
d
\end{array}\right) \cdot\left(\begin{array}{c}
d-1 \\
d-r-1
\end{array}\right) \cdot\left(\begin{array}{c}
\frac{d-1}{2}+r-m+l \\
d
\end{array}\right) .
$$

This shows 1.

2. Consider $\alpha$ of the form $\alpha=\theta_{j}-\theta_{k}$ for some $1 \leq j<k \leq d$. We have already shown in the first part that $\left\langle\delta_{+}, \alpha\right\rangle=2(k-j)$. Let us denote by $v_{+}$the first component and $v$ the $d-1$ components of the highest weight corresponding to the second family of eigenvalues. We have

$$
\langle\lambda, \alpha\rangle=2\left(v_{+}, v, \ldots, v\right) \cdot\left(\begin{array}{c}
0 \\
\vdots \\
0 \\
1 \\
0 \\
\vdots \\
0 \\
-1 \\
0 \\
\vdots \\
0
\end{array}\right)
$$

Choosing $\alpha=\theta_{j}+\sum_{k=1}^{d} \theta_{k}$ with $j \in\{1, \ldots, d\}$, we already know that $\left\langle\delta_{+}, \alpha\right\rangle=2(d-j+1)$. Moreover,

$$
\begin{aligned}
\langle\lambda, \alpha\rangle=2\left(v_{+}, v, \ldots, v\right) \cdot\left(\begin{array}{c}
0 \\
\vdots \\
0 \\
1 \\
0 \\
\vdots \\
0
\end{array}\right) \\
=\mid \begin{array}{lll}
2(v+l) & \text { case } & j=1 \\
2 v & \text { case } & j>1 .
\end{array}
\end{aligned}
$$

Hence the product is given by

$$
\prod_{\alpha \in \Delta_{+}}\left(1+\frac{\langle\lambda, \alpha\rangle}{\left\langle\delta_{+}, \alpha\right\rangle}\right)=\prod_{k=2}^{d}\left(1+\frac{l}{k-1}\right) \cdot\left(1+\frac{v+l}{d}\right) \cdot \prod_{j=2}^{d}\left(1+\frac{v}{d-j+1}\right) .
$$

Of course only the central factor appears in case $d=1$. Replacing $v$ by its respective value gives 2 . and 3 . and concludes the proof.

As a consequence of Lemma 4.5 and Lemma 4.6, we obtain the

Theorem 4.7 Let $d$ be a positive odd integer and $m \in \mathbb{Z}$ be arbitrary. Denote by $\gamma_{d}$ the tautological bundle of $\mathbb{C P}^{d}$. Then the spectrum of the square of the Dirac operator of $\mathbb{C P}^{d}$ twisted with $\gamma_{d}^{m}$ is given by the following family of eigenvalues:

1. $2(r+l) \cdot(d+1+2(l-m-\epsilon))$, where $r \in\{1, \ldots, d-1\}, \epsilon \in\{0,1\}$ and $l \in \mathbb{N}$ with $l \geq \max \left(\epsilon, \frac{d+1}{2}-r+m\right)$. The multiplicity of the eigenvalue corresponding to the choice of a triple $(r, \epsilon, l)$ as above is given by

$$
\frac{d\left(\frac{d+1}{2}+r-m+2 l-\epsilon\right)}{(r+l)\left(\frac{d+1}{2}-m+l-\epsilon\right)} \cdot\left(\begin{array}{c}
d+l-\epsilon \\
d
\end{array}\right) \cdot\left(\begin{array}{c}
d-1 \\
d-r-\epsilon
\end{array}\right) \cdot\left(\begin{array}{c}
\frac{d-1}{2}+r-m+l \\
d
\end{array}\right) .
$$


2. $2 l(2 l+d-1-2 m)$, where $l \in \mathbb{N}, l \geq \max \left(0, m+\frac{d+1}{2}\right)$, with multiplicity

$$
\prod_{k=2}^{d}\left(1+\frac{l}{k-1}\right) \cdot\left(1+\frac{2 l-\frac{d+1}{2}-m}{d}\right) \cdot \prod_{j=2}^{d}\left(1+\frac{l-\frac{d+1}{2}-m}{d-j+1}\right) .
$$

3. $2(d+l)(d+1+2(l-m))$, where $l \in \mathbb{N}, l \geq \max \left(0, m-\frac{d+1}{2}\right)$, with multiplicity

$$
\prod_{k=2}^{d}\left(1+\frac{l}{k-1}\right) \cdot\left(1+\frac{2 l+\frac{d+1}{2}-m}{d}\right) \cdot \prod_{j=2}^{d}\left(1+\frac{l+\frac{d+1}{2}-m}{d-j+1}\right) .
$$

Note that, since $\mathbb{C P}^{d}$ is a symmetric space, the spectrum of every Dirac operator twisted with a homogeneous bundle over $\mathbb{C P}^{d}$ is symmetric about the origin. Hence the spectrum of the Dirac operator of $\mathbb{C P}^{d}$ twisted with $\gamma_{d}^{m}$ can be easily deduced from that of its square.

We point out that the computations done by M. Ben Halima in [3, Thm. 1] contain a minor mistake (his $m$ should be replaced by $-m$ ). It can be also checked that, up to a factor $4(d+1)$ (his convention for the Fubini-Study metric is different from ours), our values coincide with his (his $k$ is our $l$ and his $l$ is our $d-r$ ).

We can now formulate the

Theorem 4.8 Let $d<n$ be positive odd integers. Then the spectrum of the square of the Dirac operator of $\mathbb{C P}^{d}$ twisted with the spinor bundle of the normal bundle of the canonical embedding $\mathbb{C P}^{d} \rightarrow \mathbb{C P}^{n}$ is given by the following family of eigenvalues:

1. $2(r+l) \cdot(2 d+1-n+2(s+l-\epsilon))$, where $r \in\{1, \ldots, d-1\}, s \in\{0, \ldots, n-d\}, \epsilon \in\{0,1\}$ and $l \in \mathbb{N}$ with $l \geq \max \left(\epsilon, \frac{n+1}{2}-r-s\right)$. The multiplicity of the eigenvalue corresponding to the choice of a 4-tuple $(r, s, \epsilon, l)$ as above is given by

$$
\frac{d\left(d-\frac{n-1}{2}+r+s+2 l-\epsilon\right)}{(r+l)\left(d-\frac{n-1}{2}+s+l-\epsilon\right)} \cdot\left(\begin{array}{c}
n-d \\
s
\end{array}\right) \cdot\left(\begin{array}{c}
d+l-\epsilon \\
d
\end{array}\right) \cdot\left(\begin{array}{c}
d-1 \\
d-r-\epsilon
\end{array}\right) \cdot\left(\begin{array}{c}
d-\frac{n+1}{2}+r+s+l \\
d
\end{array}\right) .
$$

2. $4 l\left(l+s+d-\frac{n+1}{2}\right)$, where $s \in\{0, \ldots, n-d\}, l \in \mathbb{N}, l \geq \max \left(0, \frac{n+1}{2}-s\right)$, with multiplicity

$$
\left(\begin{array}{c}
n-d \\
s
\end{array}\right) \cdot \prod_{k=2}^{d}\left(1+\frac{l}{k-1}\right) \cdot\left(1+\frac{2 l-\frac{n+1}{2}+s}{d}\right) \cdot \prod_{j=2}^{d}\left(1+\frac{l-\frac{n+1}{2}+s}{d-j+1}\right) .
$$

3. $2(d+l)(2 d-n+1+2(l+s))$, where $s \in\{0, \ldots, n-d\}, l \in \mathbb{N}, l \geq \max \left(0, \frac{n-1}{2}-d-s\right)$, with multiplicity

$$
\left(\begin{array}{c}
n-d \\
s
\end{array}\right) \cdot \prod_{k=2}^{d}\left(1+\frac{l}{k-1}\right) \cdot\left(1+\frac{2 l+d-\frac{n-1}{2}+s}{d}\right) \cdot \prod_{j=2}^{d}\left(1+\frac{l+d-\frac{n-1}{2}+s}{d-j+1}\right) .
$$

Proof: Recall that, by Corollary 4.4, there exists a unitary and parallel isomorphism

$$
\Sigma\left(T \mathbb{C P}^{d}\right) \otimes \Sigma\left(T^{\perp} \mathbb{C P}^{d}\right) \cong \bigoplus_{s=0}^{n-d}\left(\begin{array}{c}
n-d \\
s
\end{array}\right) \cdot \Sigma\left(T \mathbb{C P}^{d}\right) \otimes \gamma_{d}^{\frac{n-d}{2}-s},
$$

where $\gamma_{d}$ is the tautological bundle of $\mathbb{C P}^{d}$ and $\left(\begin{array}{c}n-d \\ s\end{array}\right)$ stands for the multiplicity with which the subbundle $\Sigma\left(T \mathbb{C P}^{d}\right) \otimes \gamma_{d}^{\frac{n-d}{2}-s}$ appears in the splitting. Therefore, the eigenvalues of the twisted Dirac operator acting on $\Sigma\left(T \mathbb{C P}^{d}\right) \otimes \Sigma\left(T^{\perp} \mathbb{C P}^{d}\right)$ are those of $\Sigma\left(T \mathbb{C P}^{d}\right) \otimes \gamma_{d}^{\frac{n-d}{2}-s}$, where $s$ runs from 0 to $n-d$. Moreover, the multiplicity of the eigenvalue corresponding to some $s$ is $\left(\begin{array}{c}n-d \\ s\end{array}\right)$ times the multiplicity 
computed in Lemma 4.6. Replacing $m$ by $\frac{n-d}{2}-s$, Theorem 4.7 gives the result.

Note that $(d+1)^{2}$ is always an eigenvalue for the squared operator $\left(D_{M}^{\Sigma N}\right)^{2}:$ if $d=1$, take $s=\frac{n-1}{2}$ and $l=1$ in the second family of eigenvalues; if $d>1$, take $r=\frac{d+1}{2}, s=\frac{n-d}{2}$ and $\epsilon=0=l$ in the first family.

Using Theorem 4.8, we are now able to compute the smallest eigenvalue of the twisted Dirac operator:

Proposition 4.9 The lowest eigenvalue for the square of the Dirac operator of $\mathbb{C P}^{d}$ twisted with the spinor bundle of the normal bundle of the canonical embedding $\mathbb{C P}^{d} \rightarrow \mathbb{C P}^{n}$ is equal to 0 for $d<\frac{n+1}{2}$ and to $(n+1)(2 d+1-n)$ for $d \geq \frac{n+1}{2}$.

Proof: Let us consider the first family of eigenvalues with $\epsilon=0$ (the same computation remains true for $\epsilon=1)$. For $r+s \geq \frac{n+1}{2}$, which implies $d-\frac{n-1}{2} \leq r$, the minimum is attained for $l=0$ and we find the eigenvalues $2 r(2 d+1-n+2 s)$, which are increasing functions with respect to $s$ with $s \geq \frac{n+1}{2}-r$. Here two cases occur:

1. Case where $\frac{n+1}{2}-r \geq 0$, the eigenvalues become $4 r(d+1-r)$ and we distinguish the two subcases:

(a) For $d \leq \frac{n+1}{2}$, then the lowest eigenvalue is equal to $4 d$.

(b) For $\frac{n+1}{2}<d$, the lowest eigenvalue is $(n+1)(2 d+1-n)$.

2. Case where $\frac{n+1}{2}-r<0$ which implies $\frac{n+1}{2}<d$. Hence, the lowest eigenvalue is equal to $(n+$ 1) $(2 d+1-n)$.

Now for $r+s<\frac{n+1}{2}$, we take $l=\frac{n+1}{2}-r-s$. Thus the eigenvalues are equal $2(n+1-2 s)(d+1-r)$ which are decreasing functions in $s$ with $0 \leq s \leq \frac{n-1}{2}-r$. We have:

1. Case where $\frac{n-1}{2}-r \leq n-d$. We then get the eigenvalues $4(1+r)(d+1-r)$ with $d-\frac{n+1}{2} \leq r \leq \frac{n-1}{2}$. Here two cases occur:

(a) For $d \leq \frac{n+1}{2}$, the lowest eigenvalue is equal to $8 d$.

(b) For $d>\frac{n+1}{2}$, the lowest eigenvalue is equal to $(n+3)(2 d+1-n)$.

2. Case where $\frac{n-1}{2}-r>n-d$, we get the eigenvalues $2(2 d-n+1)(d+1-r)$ with $1 \leq r \leq d-\frac{n+3}{2}$. In this case, we have that $d>\frac{n+1}{2}$ and the lowest eigenvalue is equal to $(n+5)(2 d-n+1)$.

For the second family of eigenvalues, we distinguish the cases:

1. Case where $\frac{n+1}{2}-s \leq 0$ which implies that $d \leq \frac{n-1}{2}$, we take $l=0$. The lowest eigenvalue is then equal to 0 .

2. Case where $\frac{n+1}{2}-s>0$. The eigenvalues become $2 d(n+1-2 s)$ with $0 \leq s \leq \frac{n-1}{2}$. Two cases occur

(a) For $d \leq \frac{n+1}{2}$, the lowest eigenvalue is $4 d$.

(b) For $d>\frac{n+1}{2}$, the lowest eigenvalue is $2 d(2 d+1-n)$

For the last family of eigenvalues, we consider the two cases:

1. Case where $\frac{n-1}{2}-d-s>0$, which implies that $d<\frac{n-1}{2}$, we take $l=\frac{n-1}{2}-d-s$. We find the lowest eigenvalue 0 after substituting.

2. Case where $\frac{n-1}{2}-d-s \leq 0$. In this case $l=0$ and we get $2 d(2 d-n+1+2 s)$. Here two cases occur:

(a) For $d>\frac{n-1}{2}$, the lowest eigenvalue is $2 d(2 d-n+1)$.

(b) For $d \leq \frac{n-1}{2}$, the lowest eigenvalue is 0 . 
Next we show that the estimate (2.7) is not always sharp. We consider the simplest case where $d=1$ and compare the multiplicities of the eigenvalues 0 and 4 with $2\left(\begin{array}{c}n \\ \frac{n+1}{2}\end{array}\right)$, which is the a priori number of eigenvalues bounded by 4 in (2.7). The multiplicity of the eigenvalue 0 is equal to

$$
\sum_{s=0}^{\frac{n-3}{2}}\left(\begin{array}{c}
n-1 \\
s
\end{array}\right)\left(\frac{n-1}{2}-s\right)+\sum_{s=\frac{n+1}{2}}^{n-1}\left(\begin{array}{c}
n-1 \\
s
\end{array}\right)\left(s-\frac{n-1}{2}\right),
$$

which is equal to $\sum_{s=0}^{\frac{n-3}{2}}\left(\begin{array}{c}n-1 \\ s\end{array}\right)(n-1-2 s)$ since by replacing $s$ by $(n-1)-s$ the second sum is equal to the first one. A short computation gives $\sum_{s=0}^{\frac{n-3}{2}}\left(\begin{array}{c}n-1 \\ s\end{array}\right)(n-1-2 s)=\frac{n-1}{2} \cdot\left(\begin{array}{c}n-1 \\ \frac{n-1}{2}\end{array}\right)$. On the other hand, the multiplicity of the eigenvalue 4 is equal to $4\left(\begin{array}{c}n \\ \frac{n-1}{2}\end{array}\right)$. Hence the sum of these two multiplicities is $\left(\frac{n-1}{2}+4\right) \cdot\left(\begin{array}{c}n-1 \\ \frac{n-1}{2}\end{array}\right)$. That number is always greater than $2\left(\begin{array}{c}n \\ \frac{n+1}{2}\end{array}\right)$. However, if the multiplicity of the eigenvalue 0 is smaller than $2\left(\begin{array}{c}n \\ \frac{n+1}{2}\end{array}\right)$ for $n=3,5,7$, it is greater for $n \geq 9$. Thus, the equality in (2.7) is optimal for $n=3,5,7$ but is never optimal as soon as $n \geq 9$. In particular, the twisted Dirac operator on Kähler submanifolds behaves very differently from that on submanifolds immersed in real spaceforms, where analogous upper bounds are sharp in any dimension.

\section{References}

[1] C. Bär, Extrinsic bounds for eigenvalues of the Dirac operator, Ann. Glob. Anal. Geom. 16 (1998), no. $2,573-596$.

[2] H. Baum, Eigenvalue estimates for Dirac operators coupled to instantons, Ann. Glob. Anal. Geom. 12 (1994), no. 2, 193-209.

[3] M. Ben Halima, Spectrum of twisted Dirac operators on the complex projective space $\mathbb{P}^{2 q+1}(\mathbb{C})$, Comment. Math. Univ. Carolin. 49 (2008), no. 3, 437-445.

[4] A. Besse, Einstein manifolds, Ergebnisse der Mathematik und ihrer Grenzgebiete, 3. Folge, Band 10, Springer-Verlag, Berlin, 1987.

[5] J.-P. Bourguignon, O. Hijazi, J.-L. Milhorat and A. Moroianu A spinorial approach to Riemannian and conformal geometry

[6] N. Ginoux, Opérateurs de Dirac sur les sous-variétés, PhD thesis, Université Henri Poincaré, Nancy (2002).

[7] N. Ginoux, Dirac operators on Lagrangian manifolds, J. Geom. Phys. 52 (2004), no. 4, 480-498.

[8] N. Ginoux, G. Habib and U. Semmelmann, Imaginary Kählerian Killing spinors, in preparation.

[9] N. Ginoux and B. Morel, On eigenvalue estimates for the submanifold Dirac operator, Internat. J. Math. 13 (2002), no. 5, 533-548.

[10] K.-D. Kirchberg, Killing spinors on Kähler manifolds, Ann. Global Anal. Geom. 11 (1993), no. 2, 141-164.

[11] H.B. Lawson and M.-L. Michelsohn, Spin geometry, Princeton University Press, Princeton, 1989.

[12] A. Moroianu, La première valeur propre de l'opérateur de Dirac sur les variétés kähleriennes compactes, Commun. Math. Phys. 169 (1995), 373-384. 